\section{Biobanking and biomolecular resource research infrastructure: The Italian node}

\author{
Barbara Parodi \\ Biological Resource Centre, IRCCS \\ Ospedale Policlinico San Martino (CRB- \\ HSM), Genoa, Italy
}

\begin{abstract}
BBMRI-ERIC is a European research infrastructure for biobanking, bringing together all the main players from the biobanking field - researchers, biobankers, industry, and patients - to boost biomedical research. To that end, it offers quality management services, support with ethical, legal and societal issues, and a number of online tools and software solutions. Ultimately, the goal of BBMRI-ERIC and its National Nodes is to make new treatments possible.
\end{abstract}

\section{Introduction}

Biological samples of human origin (cells and tissues) and their use in in vitro systems can be a valid alternative to animal testing in many applications in biomedical and biotechnological research. A prerequisite for the reliability of in vitro systems is the quality of samples and data. Biobanks and Biological Resource Centers can guarantee quality of samples and data, harmonization, respect for the rights of participants.

The European Strategy Forum for Research Infrastructure (ESFRI) included the BioBanking and BioMolecular Resources Research Infrastructure in the 2006 Roadmap, among the six strategic research infrastructures for Europe in the field of Life Sciences.

\section{Results}

BBMRI-ERIC, the European research infrastructure for biobanking, was set up in 2013 and brings together all the main players from the biobanking field - researchers, biobankers, industry, and patients - to boost biomedical research. To that end, it offers quality management services, support with ethical, legal and societal issues, and a number of online tools and software solutions. Ultimately, the goal of BBMRI-ERIC and its Nodes is to make new treatments possible. The principles behind BBMRI-ERIC are the scientific excellence and the European relevance of the participating institutions, the long-term perspective of biobanking projects, the transparency of the aims, an efficient and flexible organization, the enhancement of the existing resources in terms of structures and skills.

BBMRI.it, the National Node of BBMRI-ERIC, was set up thanks to the joint commitment of the Ministry of Health and the Ministry of University and Research. Istituto Superiore di Sanità, Consiglio Nazionale della Ricerca, 33 Scientific Institutes of Hospitalization and Care (IRCCS), 19 Universities, 45 Hospitals, and a network of stakeholders
Correspondence: Barbara Parodi, Biological Resource Centre, IRCCS Ospedale Policlinico San Martino (CRB-HSM), Genoa, Italy.

E-mail: barbara.parodi@hsanmartino.it

Key words: Biobanking; infrastructure; biological samples.

Conference presentation: this paper was presented at the Second Centro 3R Annual Meeting - 3Rs in Italian Universities, 2019, June 20-21, University of Genoa, Italy.

Received for publication: 28 October 2019. Accepted for publication: 6 November 2019.

This work is licensed under a Creative Commons Attribution NonCommercial 4.0 License (CC BY-NC 4.0).

CCopyright: the Author(s), 2019

Licensee PAGEPress, Italy

Biomedical Science and Engineering 2019; 3(s2):100 doi:10.4081/bse.2019.100

participate in the infrastructure, including 8 patient associations including UNIAMO, (Italian Federation of Rare Diseases) and FAVO (Italian Federation of Voluntary Associations in Oncology), companies in the biomedical and biotechnological fields and scientific societies. BBMRI.it is a distributed infrastructure that includes more than 90 Biobanks, Biological Resource Centers and Collections located in different Italian regions and three Common Services (CS Quality management, CS Information Technology, CS ELSI for ethical, legal and social issues). 